\title{
Regulation statt Substitution - Biochemie nach Dr. Schüßler
}

\author{
„Ich möchte mein Kind aber nicht an \\ die Einnahme von Medikamenten \\ gewöhnen!" Wie oft hören das \\ Behandler in ihrer Praxis. Bei der \\ Anwendung der Biochemie nach \\ Dr. Schüßler ist hier jedoch eine \\ Ausnahme zu beobachten: Die meisten \\ Eltern haben keinerlei Probleme, diese \\ Behandlungsform für ihre Kinder zu \\ akzeptieren. Das Verabreichen von \\ Schüßler-Salzen wird nicht als Medika- \\ tion empfunden - berechtigterweise!
}

Sanfte Therapieformen, die ohne den Einsatz von chemischen Präparaten oder starken Medikamenten auskommen, haben sich in der Kinderheilkunde besonders bewährt. So auch die Biochemie nach Dr. Schüßler. Unabhängig von der verwendeten Potenzierung ist diese Methode keine Substitutionstherapie, sondern eine Regulationstherapie. Gerade junge Menschen reagieren auf therapeutische Reize im feinstofflichen Bereich i.d. R. mit der gewünschten Reizantwort, die die Eigenregulation des Patienten nach Möglichkeit wieder in Gang setzt.

Auf der Ebene der Mineralstoffe ist die Biochemie nach Dr. Schüßler eine große Hilfe bei diesem Prozess. Basierend auf den Arbeiten von Dr. Schüßler wird auf die 12 wichtigsten Mineralsalze des Körpers Einfluss genommen in Bezug auf ihre Aufnahme und Ausscheidung, ihre Verteilung im Körper und ihre Einbindung in die Stoffwechselabläufe.

Da bei der Biochemie nach Dr. Schüßler nur die 12 wichtigsten Mineralverbindungen betrachtet werden, ist diese Behandlungsform sehr übersichtlich und gut anwendbar ( Kasten, S. 57).

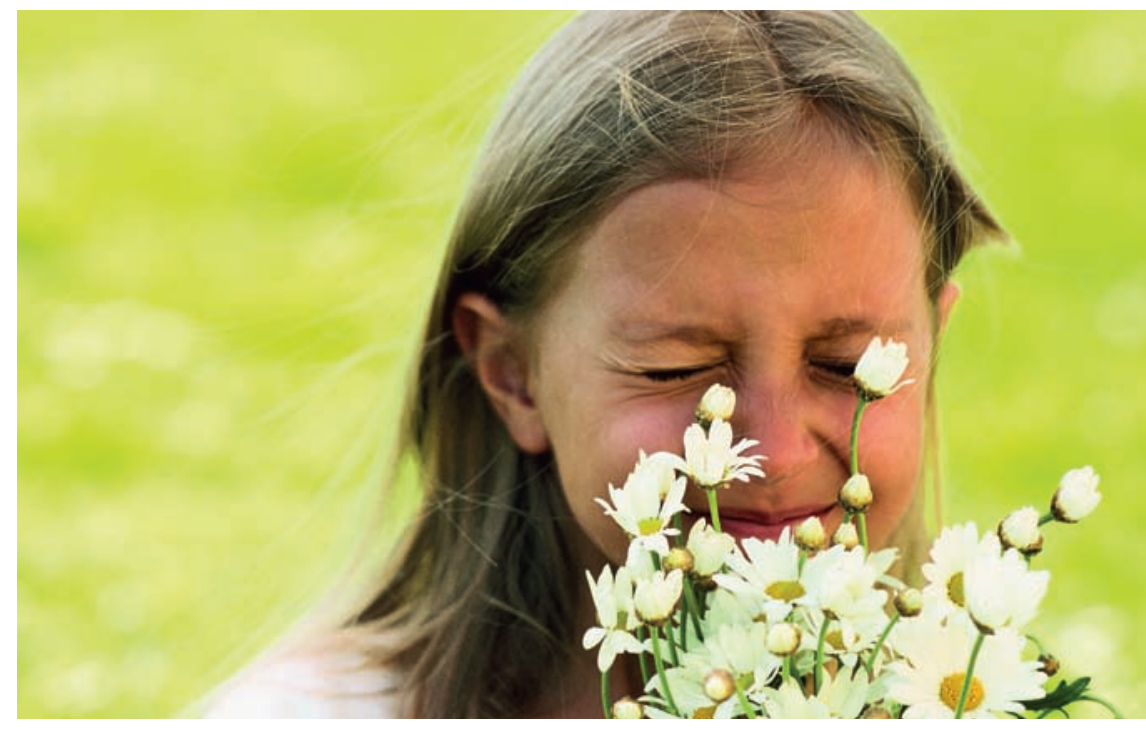

Abb. 1 Ob bei Allergien oder anderen Beschwerden: Die Biochemie nach Dr. Schüßler hat sich in der Kinderheilkunde bewährt. Foto: ๑ MEV/Bauer Roland

\section{Anwendungsbeispiele}

Rechtzeitig eingesetzt, kann die Biochemie nach Dr. Schüßler in vielen Fällen die Ausprägung einer Erkrankung abwenden oder eine problemlosere Abheilung von Krankheiten und damit schnellere Wiederherstellung der Gesundheit begünstigen. Dabei führen die Symptome einer Erkrankung den Behandler zu den richtigen Mitteln. Hier einige Beispiele für die Behandlung von Erkrankungen und Beschwerden, die häufig bei Kindern vorkommen.

\section{Allergische Reaktionen}

- Nr. 2 Calcium phosphoricum D 6: Senken der Überempfindlichkeit, Abgrenzung

- Nr. 3 Ferrum phosphoricum D 12: Entzündung (Rötung, Schwellung)
- Nr. 4 Kalium chloratum D 6: Rötung der Augen und Bindehäute

- Nr. 8 Natrium chloratum D 6: ödematöse Schwellung, tränende Augen, laufende Nase

\section{Infekte}

Bei der Anwendung der Schüßler-Salze für die Behandlung eines Infekts braucht es etwas Kreativität, da kein Infekt genau wie der andere abläuft und die Mittelwahl von Fall zu Fall variieren kann. Zu Beginn der Anwendung: 1- bis 2-mal eine „Heiße Drei“, danach häufige Gabe vom Nr. 3 Ferrum phosphoricum. Nach Bedarf 1-2 Mittel dazugeben, die Mittel zusammen in einem Schälchen an das Krankenbett stellen. Das Kind soll die Tabletten oder Globuli „wie Bonbons lutschen“. Dabei ist darauf zu achten, dass es die Mittel auch 
wirklich einnimmt. Wichtig: ausreichende Flüssigkeitszufuhr! Die Schüßler-Salze können auch in Wasser oder Tee aufgelöst und getrunken werden. Bei Bedarf ist die Zusammenstellung der Mittel zu ändern.

\section{Beispielanwendung:}

- Nr. 3 Ferrum phosphoricum D 12:

1. Entzündungsstadium: Fieber bis $39^{\circ} \mathrm{C}$. Versuch, den Infekt mit einigen Anwendungen der „Heißen Drei“ abzuwenden

- Nr. 10 Natrium sulfuricum D 6: Patient kann nicht schwitzen

- Nr. 4 Kalium chloratum D 6:

2. Entzündungsstadium: Nase beginnt zu laufen, evtl. bilden sich Borken

- Nr. 5 Kalium phosphoricum D 6: höheres Fieber (über $39^{\circ} \mathrm{C}$ ), kräftezehrend

- Nr. 6 Kalium sulfuricum D 6:

3. Entzündungsstadium: Fieber sinkt, geschädigte Schleimhaut wird Noch ca. 10 Tage über die als abgeschlossen empfundene Gesundung hinaus 5 Tbl. tgl. einnehmen.

\section{Husten}

Husten ist nicht gleich Husten! Die erfolgreiche Behandlung eines Hustens bedarf der Differenzierung. Die Entwicklung der Erkrankung muss bei der Mittelwahl beachtet werden. abgestoßen, Regenerationsstadium.

Ebenso ist zu bedenken, dass der Husten meist nicht von der Entstehung bis zum Abklingen mit ein und demselben Mittel behandelt werden kann.

Nr. 2 Calcium phosphoricum D 6: Der Schleim ist glasig, die Bronchien fühlen sich wund an

- Nr. 3 Ferrum phosphoricum D 12: schmerzende Schleimhaut, trockener Reizhusten. Meist zu Beginn des Infekts

- Nr. 4 Kalium chloratum D 6: Der Schleim löst sich schwer, zieht Fäden

- Nr. 6 Kalium sulfuricum D 6: gelb-grünlicher Schleim, die bakterielle Infektion des ursprünglich viralen Infektes. Stellt keine Komplikation dar, sondern ist normaler Verlauf des Infektes. Beginn der Heilung und Regeneration

- Nr. 7 Magnesium phosphoricum D 6: Der Husten ist krampfartig, richtiges Durchhusten ist erschwert

- Nr. 8 Natrium chloratum D 6: Die Schleimhäute sind trocken, Reiz- und Kitzelhusten

- Nr. 10 Natrium sulfuricum D 6: zäher, grünlich-gelber Auswurf

- Nr. 11 Silicea D 12: dauerndes Hüsteln, Kräftigung der Schleimhaut

Die Schüßler-Salze und ihre Anwendungsgebiete

- Nr. 1 Calcium fluoratum D 12: Elastizitätsmittel. Festigt, was zu weich ist, und macht elastisch, was zu fest ist.

- Nr. 2 Calcium phosphoricum D 6: Aufbau- und Erhaltungsmittel. Festigt Grenzstrukturen, Zellerhaltungsmittel

- Nr. 3 Ferrum phopsphoricum D 12: Tonisierungsmittel, Fiebermittel. Reguliert Eisenverwertung, Mittel bei beginnenden Infekten

- Nr. 4 Kalium chloratum D 6: Lymphmittel. Hält Fibrin in Lösung, vermeidet Verkrustungen

- Nr. 5 Kalium phosphoricum D 6: Nierenmittel. Energie für jede Zelle, Mittel bei höherem Fieber

- Nr. 6 Kalium sulfuricum D 6: Lebermittel, Stoffwechselmittel,
Reinigungsmittel nach Entzündungen

- Nr. 7 Magnesium phosphoricum D 6: Krampfmittel, Nervenmittel, stärkt das Lymphsystem

- Nr. 8 Natrium chloratum D 6: Wasserverteilungsmittel, reguliert Salz- und Flüssigkeitshaushalt

- Nr. 9 Natrium phosphoricum D 6: Säuremittel, Fettstoffwechselmittel, hilft Säuren auszuscheiden

- Nr. 10 Natrium sulfuricum D 6: Ausleitungsmittel, unterstützt die Ausscheidungsorgane

- Nr. 11 Silicea D 12: Bindegewebsmittel, festigt und entschlackt

- Nr. 12 Calcium sulfuricum D 6: „)oker“: Reaktionsmittel bei trägen Reizantworten. Aktiviert Eiterherde, ausscheidungsfördernde Wirkung auf Schleimhäute

\section{Schnupfen}

- Nr. 3 Ferrum phosphoricum D 12:

1. Entzündungsstadium: Fieber bis ca. $39^{\circ} \mathrm{C}$, Beginn der Infektion. Nase ist heiß, rot, geschwollen, schmerzhaft

- Nr. 4 Kalium chloratum D 6:

2. Entzündungsstadium: Schleimhäute sind geschwollen, Schleim wird vermehrt gebildet

- Nr. 5 Kalium phosphoricum D 6: höheres Fieber (über $39^{\circ} \mathrm{C}$ ), Abgeschlagenheit

- Nr. 6 Kalium sulfuricum D 6: 3. Entzündungsstadium: gelb-grünlicher Schleim, Beginn der Regeneration, Abstoßung verbrauchter Schleimhautstrukturen

- Nr. 8 Natrium chloratum D 6: Fließschnupfen, Stockschnupfen, geschwollene Schleimhäute, tränende Augen

- Nr. 12 Calcium sulfuricum D 6: hilft, festsitzenden Schleim abzustoßen, zwischendurch als Reaktionsmittel

\section{Hautprobleme}

Erkrankungen der Haut sind gerade bei Kindern häufig. Die Biochemie nach $\mathrm{Dr}$. Schüßler ist in der Lage, dieses komplexe Organ in all seinen vielfältigen Funktionen zu unterstützen und Störungen, die sich auf die Haut auswirken, zu regulieren.

Die Schüßler-Salze können neben der Einnahme auch äußerlich angewandt werden: einige Tbl. mit etwas Wasser (oder Tee, z. B. aus Gänseblümchen) zu einem Brei verrühren und auf die betroffenen Bereiche streichen. Zur Entsäuerung und Entgiftung kann dem Brei auch etwas Heilerde beigefügt werden. Ebenso ist die Mischung der Schüßler-Salze mit einer neutralen Salben- bzw. Cremegrundlage möglich, womit die betroffenen Hautstellen behandelt werden.

\section{Nässende Hauterkrankungen}

Bei nässenden Erkrankungen der Haut kann die Erscheinungsform der abgegebenen Flüssigkeit den Behandler zum richtigen Mittel führen. Dabei ist es unerheblich, ob die Flüssigkeit in Bläschen eingeschlossen ist oder aus der Haut austritt. Wichtig: Die Flüssigkeit inspizieren, bevor sie eingetrocknet ist!

- Nr. 8 Natrium chloratum D 6: klare Flüssigkeit, farblos wie Wasser 


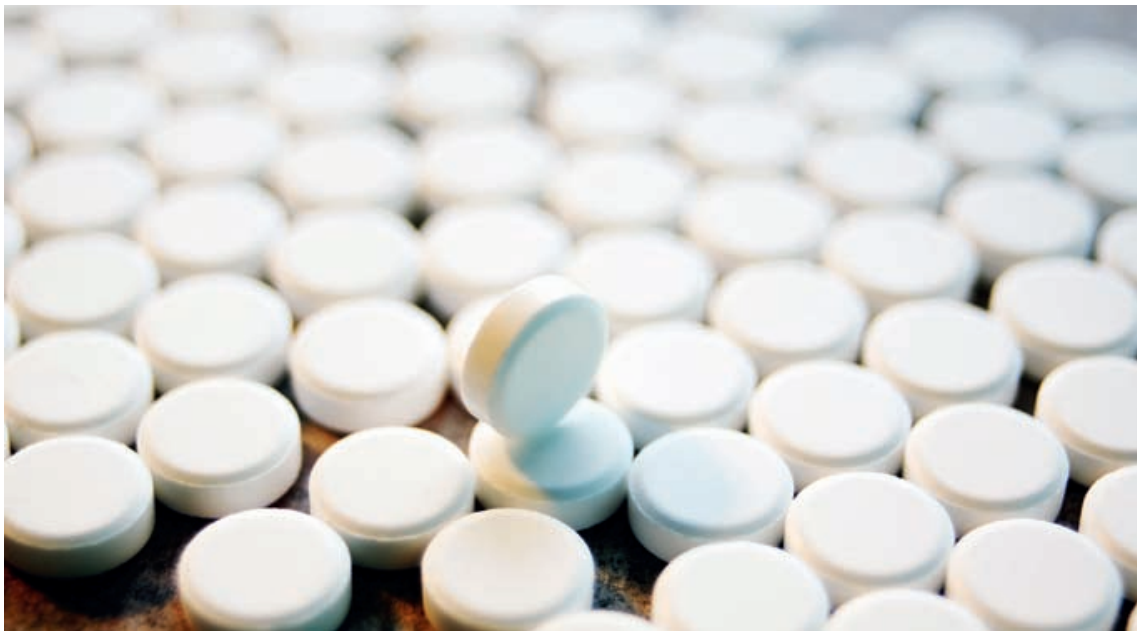

Abb. 2 Meist werden die Schüßler-Salze in Form von Tabletten eingesetzt. Foto: (c) Thieme Verlagsgruppe/Chris Meier

- Nr. 9 Natrium phosphoricum D 6: schleimige Flüssigkeit, gelblich gefärbt

- Nr. 10 Natrium sulfuricum D 6: klare Flüssigkeit, gelblich gefärbt

\section{Hauterkrankungen mit Krusten/ Borken}

- Nr. 1 Calcium fluoratum D 12: Einrisse, Fissuren, Verhärtungen. Harte Krusten, die weitere Einrisse verursachen

- Nr. 2 Calcium phosphoricum D 6: weißlich-gelbe Krusten

- Nr. 11 Silicea D 12: gelblich-eitrige Krusten

\section{Entzündliche Hauterkrankungen}

- Nr. 3 Ferrum phosphoricum D 12: Rötung, Schmerz

- Nr. 5 Kalium phosphoricum D 6, Nr. 8 Natrium chloratum D 6: Nesselsucht/ Nesselausschlag

- Nr. 7 Magnesium phosphoricum D 6: Juckreiz, Schmerz bei leichter Berührung, bei festerem Anfassen nicht so empfindlich

- Nr. 8 Natrium chloratum D6, Nr. 10 Natrium sulfuricum D 6: bei ödematöser Schwellung zusammen verabreichen

- Nr. 9 Natrium phosphoricum D 6, Nr. 11 Silicea D 12: bei Entzündung von Talgdrüsen zusammen verabreichen

\section{Bindegewebsstärkung}

- Nr. 1 Calcium fluoratum D 12: Einschleusen von Kalzium, Elastizität
- Nr. 2 Calcium phosphoricum D 6: Einschleusen von Kalzium, Stabilisieren der Zellmembran

- Nr. 11 Silicea D 12: Einschleusen von Kieselsäure, elastische Festigkeit

\section{Energielosigkeit}

- Nr. 3 Ferrum phosphoricum D 12: Verbesserung von Sauerstofftransport und -verwertung

- Nr. 5 Kalium phosphoricum D 6: Energie für jede Zelle

- Nr. 7 Magnesium phosphoricum D 6: Nervenstärkung, Coenzym

\section{Lernschwierigkeiten}

- Nr. 5 Kalium phosphoricum D 6: Energie für jede Zelle, Unterstützung von Denkprozessen

- Nr. 6 Kalium sulfuricum D 6: Verbesserung der Sauerstoffübertragung auf Zellen

- Nr. 7 Magnesium phosphoricum D 6: Coenzym, Verbesserung des Nervenzellstoffwechsels

\section{Das Mittel ist wichtiger als die Potenz}

Nun könnte man bezüglich der zu verwendenden Potenzierung der Schüßler-Salze genauso streiten wie über Dosierung und Anwendungsform - es existieren viele Meinungen und Ansichten dazu. Dr. Schüßler hat in seinem Werk „Eine abgekürzte Therapie“ sinngemäß geschrieben, dass es weitaus wichtiger ist, das richtige Mittel zu wählen als zu großes Augenmerk auf Potenzierung, Darreichungsform und Do-
Regulationstherapie versus Polypharmakologie

Im Bereich der gängigen medizinischen Versorgung steht die Behandlung - viele sagen sogar Unterdrückung - von Krankheitssymptomen im Vordergrund. Ursachen von Krankheitszeichen wird vielfach nicht auf den Grund gegangen. In vielen Fällen führt das - nicht nur im geriatrischen Bereich - zur Polypharmakologie, also zum breitbandigen Einsatz verschiedener Medikamente zur selben Zeit.

Beginnt diese Form der Therapie schon in jungen Jahren, besteht die Gefahr, dass die verwendeten Medikamente selbst eine Ursache für weitere gesundheitliche Störungen darstellen. Seit dem GKV-Modernisierungsgesetz im Jahr 2004 ist die Verschreibungspflicht von Medikamenten bei Kindern über 12 Jahren Voraussetzung für die Erstattung der Kosten durch die gesetzliche Krankenversicherung. Daraus hat sich eine aus naturheilkundlicher Sicht nur sehr schwer nachvollziehbare Situation entwickelt: Ein großer Teil der medikamentösen Versorgung der Bevölkerung erfolgt mit Arzneimitteln, die aufgrund einer möglichen Gefährdung der Patientengesundheit in die Verschreibungspflicht überführt worden sind!

sierung zu richten. Ich empfehle die Anwendung der Schüßler-Salze in der Normalpotenzierung ( Kasten, S. 57). Meiner Erfahrung nach ist es unerheblich, ob nun eine D 6 oder D 9 verwendet wird.

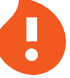

Mit einer bestimmten Potenzierung eine bestimmte Wirkung erreichen zu wollen, widerspricht dem grundsätzlichen Regulationsgedanken.

Die Verwendung verschiedener Potenzierungsstufen für differenzierte Anwendungsbereiche macht dieses so faszinierend übersichtliche System mit seinen wenigen Therapiebausteinen viel unübersichtlicher, ohne dabei therapeutisch relevante Vorteile zu bringen. 
Meist werden die Schüßler-Salze in Form von Tabletten eingesetzt, häufig auch als Globuli. Eltern greifen für ihre Kinder gerne zu dieser Darreichungsform, da es sich dabei eben nicht um die medikamententypische Tablettenform handelt.

\section{Dosierungsempfehlung}

Für die Dosierung der Schüßler-Salze empfehle ich als groben Richtwert: Bei akuten Erkrankungen, z. B. einem beginnenden grippalen Infekt, können die Mittel initial in Form der „Heißen Anwendung“ verabreicht werden, d. h. 5-10 Tbl. oder 10-20 Glob. in heißem Wasser lösen. Bei der Verwendung von Tabletten setzt sich der Milchzucker, aus dem sie größtenteils bestehen, am Boden des Glases ab und wird nicht mitgetrunken. Die „Heiße Anwendung“" wird dann schluckweise getrunken. Die durch die Wärme intensivierte Durchblutung hilft, die Informationen der Mineralsalze gut durch die Schleimhaut in das Blut aufzunehmen.

Danach wird die Einnahme mit kleinen, recht eng aufeinanderfolgenden Dosierungen fortgeführt: alle paar Minuten 1 Tbl. oder wenige Glob. im Mund zergehen lassen. Kinder haben i. d. R. ein gutes Bauchgefühl, was die Einnahme angeht. Sie können die Anwendung in Eigenregie steuern, wobei natürlich die elterliche Kontrolle nicht fehlen darf. Hier kommt ein weiterer großer Vorteil der Biochemie nach Dr. Schüßler zum Tragen: Eine Überdosie- rung der Mittel ist nicht möglich, schlimmstenfalls kann es zu Beschwerden im Bereich des Verdauungstrakts kommen, weil der aufgenommene Milchzucker aus den Tabletten zu weicherem Stuhl oder leichten Durchfällen führt. Tatsächlich ist dieser Effekt bei Kindern jedoch erst bei Dosierungen ab 50 Tbl. tgl. zu erwarten. Kinder besitzen i.d.R. aber noch ausreichend Laktase, sodass die Verdauung des Milchzuckers für sie meist kein Problem darstellt.

Bei längeren Krankheitsverläufen wird 3- bis 5-mal tgl. 1 Gabe der Mineralsalze verabreicht, also ca. 5 Tbl. oder 15 Glob. pro Tag und Schüßler-Salz.

\section{Eine häufigere und geringere Dosierung ist gegenüber einer seltener verabreichten höheren vorzuziehen.}

Grundsätzlich gilt: Nicht zu viele SchüßlerSalze auf einmal geben, 2-3 am selben Tag sollten zur Behandlung ausreichen. Dr. Schüßler favorisierte die Verabreichung nur eines Mittels pro Tag. Seine Befürchtung, sonst könnten die Mittel miteinander konkurrieren oder es könnte zu antagonistischen Effekten führen, hat sich jedoch durch die langjährig gesammelte Erfahrung mit dieser Behandlungsmethode als unbegründet herausgestellt. Dennoch gilt: Eine Reiz- und Informationsüberflutung im therapeutischen Bereich ist der Gesundung nicht dienlich!
Dieser Artikel ist online zu finden unter: http://dx.doi.org//10.1055/s-0033-1337382

\section{Verwendete Literatur}

[1] Broy J. Die Biochemie nach Dr. Schüßler. Augsburg: Foitzick; 2009

[2] Germann P, Schlutt U. Vademecum Biochemie. Ein Leitfaden zur Biochemie nach Dr. Schüßler; 2006

[3] Schüßler W. Eine abgekürzte Therapie. Oldenburg: Schulzesche Hof-Buchdruckerei; 1898

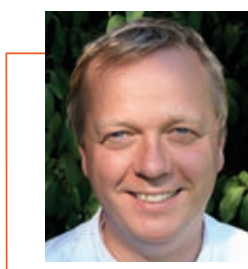

HP Uwe Schlutt Im Karrenberg 56 44329 Dortmund

Uwe Schlutt ist Heilpraktiker seit 1995. Seine Tätigkeitsschwerpunkte sind u.a.: Ausleitungs- und Umstimmungsverfahren, Phyto-Aroma-Therapie, homöopathische Komplexmittel und Biochemie nach Dr. Schüßler. Er ist lehrtätig an verschiedenen Heilpraktikerschulen und leitet Weiterbildungen im Auftrag der Apothekenkammer Westfalen-Lippe im Bereich Naturheilkunde und Homöopathie.

E-Mail:Schlutt@Das-Gesundheitshaus.de 